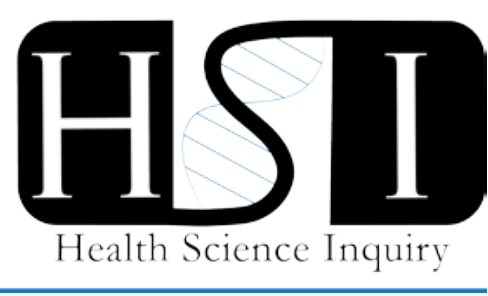

\title{
Targeting our aging cells to treat metabolic disease
}

\author{
Jessica Bertschmann \\ Department of Biochemistry and Molecular Biology, University of Calgary
}

\section{Introduction}

Aging is the leading predictor of the major chronic diseases that account for the majority of morbidity, mortality, and health costs worldwide [1]. While we cannot reverse our chronological age, we can influence our physiological aging. As our cells age and encounter stress, they become "worn out" and enter a state known as cellular senescence. The discovery of the accumulation of senescent cells in aged and damaged tissues has provided insight into one of the underlying causes of age-related pathology, and has emerged as a potential target for the treatment of chronic diseases [2]. Recently, the accumulation of senescent cells has been associated with metabolic disorders, such as type II diabetes and obesity. Pharmacological targeting of cellular senescence may have significant impact on disease pathogenesis, and could be more effective in preventing the progression of diabetes complications than currently available therapies, which have limited impact on previously existing tissue damage [3]. An emerging class of drugs, termed senolytics, selectively targets senescent cells in diseased tissues, and has shown promising results in pre-clinical trials [1].

\section{Cellular senescence and the secretory phenotype}

The cells in our body can undergo a finite number of divisions. With every round of DNA replication, the repetitive DNA sequence at the ends of chromosomes, called telomeres, become progressively shorter until they reach a critically short length that results in cellular senescence. As cells become senescent, they lose the ability to divide and take on a distinct morphology [2]. Although initially identified as a response to the progressive shortening of telomeres, senescence may also be triggered by a variety of other stressful stimuli and cellular insults. Senescence is therefore an essential mechanism for preventing the proliferation of aged and damaged cells. However, when the immune system is unable to effectively clear senescent cells, the cells accumulate and have many pathological effects [4].

Senescent cells also secrete large quantities of over 100 different soluble factors [5], collectively known as the senescence-associated secretory phenotype (SASP). These factors include inflammatory cytokines and chemokines, proteases, fibrotic factors and growth factors that disturb the tissue microenvironment. Beyond its effects on tissue function, the SASP contains factors that induce senescence in neighboring cells, setting off a cascade of events that culminates in the formation of functionally aged and/or diseased tissue. Thus, both the accumulation of senescent cells and the secretsion of pro-inflammatory factors have been shown to contribute to tissue damage and the pathology of metabolic diseases [2] (Figure 1).

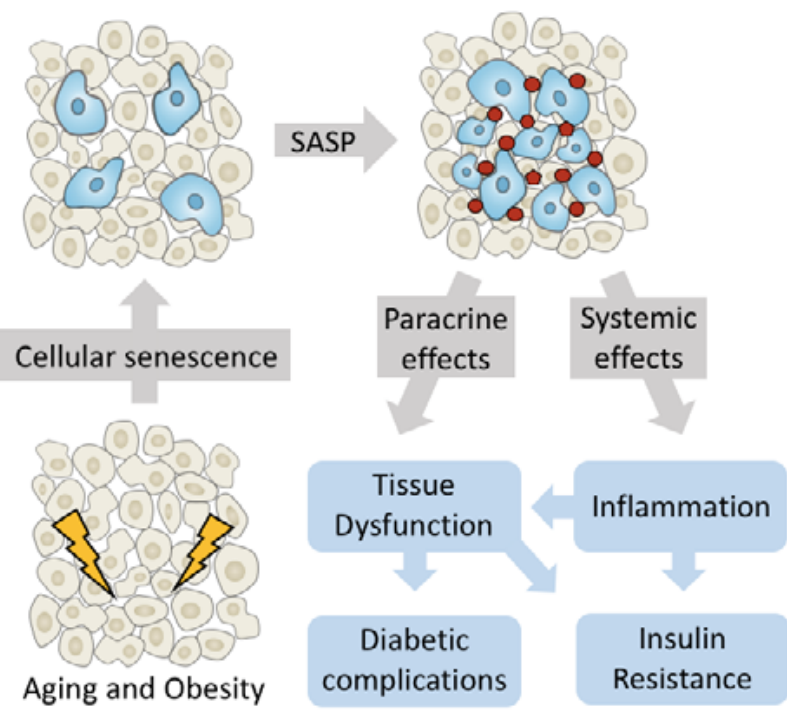

Figure 1: Pathological effects of cellular senescence and its role in driving insulin resistance and diabetes complications. Adapted from [3].

\section{The role of senescence in metabolic disease}

Cellular senescence in adipose tissues has been associated with obesity and contributes to its pathological effects. Caloric overload, due to nutritional excess or low energy expenditure, leads to the storage of energy in adipose tissue. When the storage capacity of adipocytes reaches a certain threshold, it triggers a stress response that results in cellular senescence and local inflammation. This 
senescence response initiates a cascade of events that give rise to liver steatosis and insulin resistance, two of the hallmarks of metabolic syndrome [6]. Interestingly, when mice were genetically manipulated to block adipocyte senescence, they had a lower body weight and were protected against insulin resistance induced by chronic high-fat diet [7].

Insulin resistance, due to obesity and ageing, is initially compensated by the proliferation of insulin-producing islets of beta cells. This compensatory proliferation leads to proliferative exhaustion, accelerating cellular senescence and contributing to type II diabetes [8]. In turn, metabolic and signaling changes seen in diabetes, such as high circulating glucose, altered lipid metabolism, and growth hormone axis perturbations, create a microenvironment that promotes the induction of cellular senescence. Thus, senescence is part of a diabetic pathogenic loop, acting as both a cause and a consequence of metabolic changes and tissue damage [3].

\section{Cellular senescence as an emerging therapeutic target}

The current gold-standard treatment for obesity and type 2 diabetes are glucose-lowering medications that are combined with a diet and exercise regime. However, these treatments do little to ameliorate existing tissue damage, and have a limited effect on the pathogenic loop that is established by senescent cells. Senescent cell-targeted therapies present new opportunities for targeting one of the underlying causes of type 2 diabetes and potentially reversing some of its complications [3] (Figure 2).

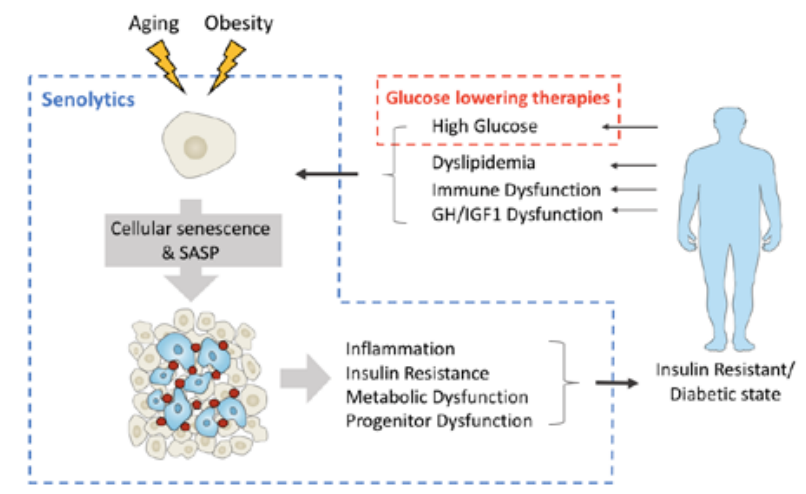

Figure 2: Senolytics present new opportunities for targeting type 2 diabetes and its complications. Adapted from [3].

Senolytics are senescent cell-clearing drugs that typically act either by selectively inducing apoptosis in senescent cells or by preventing senescent cells from secreting harmful factors [1]. Although these drugs are still in the early stages of development, they have shown promise in preclinical and clinical trials. Because cellular senescence is a basic aging mechanism that is thought to play a role in numerous age-related diseases, targeting senescent cells could have a widespread impact on individual patients and on a population-wide scale [3].

\section{References}

[1] Kirkland, J. L., Tchkonia, T., Zhu, Y., Niedernhofer, L. J., Robbins, P. D. (2017) The Clinical Potential of Senolytic Drugs. J Am Geriatr Soc, 65 (10), 22972301. Available from: http://doi.wiley.com/10.1111/jgs.14969.

[2] Muñoz-Espín, D., Serrano, M. (2014) Cellular senescence: from physiology to pathology. Nat Rev Mol Cell Biol, 15 (7), 482496. Available from: http://www.nature.com/articles/nrm3823.

[3] Palmer, A. K., Tchkonia, T., LeBrasseur, N. K., Chini, E.N., Xu, M., Kirkland, J. L (2015) Cellular Senescence in Type 2 Diabetes: A Therapeutic Opportunity. Diabetes, 64 (7), 22892298. Available from: http://www.ncbi.nlm.nih.gov/pubmed/26106186.

[4] Lecot, P., Alimirah, F., Desprez, P-Y., Campisi, J., Wiley, C. (2016) Context-dependent effects of cellular senescence in cancer development. Br J Cancer, 114 (11):11801184. Available from: http://www.nature.com/articles/bjc2016115.

[5] Coppé, J-P., Desprez, P-Y., Krtolica, A., Campisi, J. (2010) The Senescence-Associated Secretory Phenotype: The Dark Side of Tumor Suppression. Annu Rev Pathol Mech Dis, 5 (1), 99118. Available from: http://www.annualreviews.org/doi/10.1146/annurevpathol-121808-102144.

[6] Gregor, M. F., Hotamisligil, G. S. (2011) Inflammatory Mechanisms in Obesity. Annu Rev Immunol, 29 (1), 415445. Available from: http://www.annualreviews.org/doi/10.1146/annurevimmunol-031210-101322.

[7] Minamino, T., Orimo, M., Shimizu, I., Kunieda, T., Yokoyama, M., Ito, T., et al. (2009) A crucial role for adipose tissue p53 in the regulation of insulin resistance. Nat Med, 15 (9), 10821087. Available from: http://www.nature.com/articles/nm.2014.

[8] Sone, H., Kagawa, Y. (2005) Pancreatic beta cell senescence contributes to the pathogenesis of type 2 diabetes in high-fat diet-induced diabetic mice. Diabetologia, 48 (1), 5867. Available from: http://link.springer.com/10.1007/s00125-004-1605-2. 


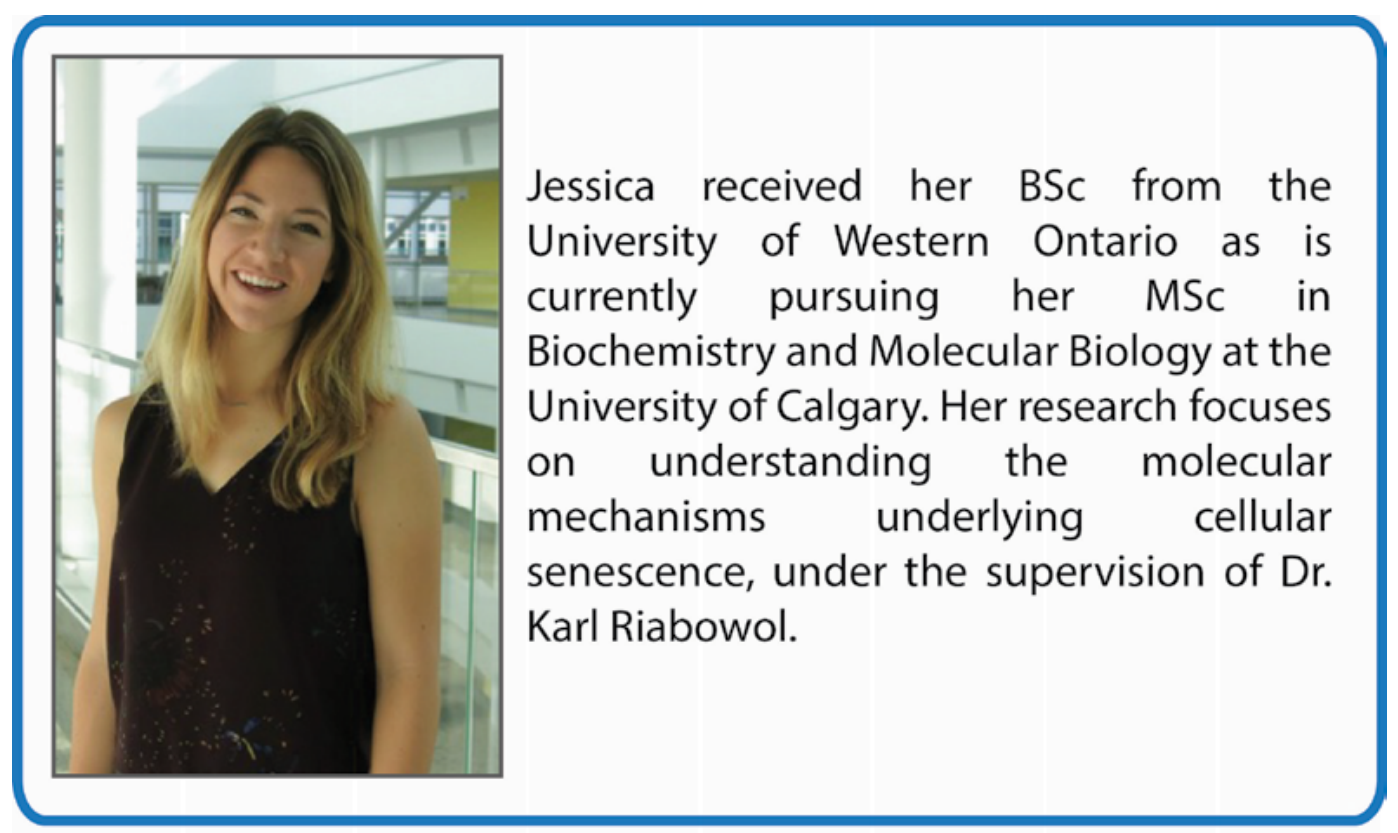

